\title{
Preservação da fertilidade em homens jovens com câncer: conceitos atuais e o futuro
}

\author{
Fertility preservation in young men with cancer: \\ current concepts and future
}

\author{
Conrado Alvarenga', Ana Paula Garcia Cardoso², Marcello S. Cocuzza', Miguel Srougi ${ }^{3}$
}

Alvarenga C, Cardoso AP, Cocuzza M, Srougi M. Preservação da fertilidade em homens jovens com câncer: conceitos atuais e o futuro / Fertility preservation in young men with cancer: current concepts and future. Rev Med (São Paulo). 2012 jul.-set.;91(3):223-8.

\begin{abstract}
RESUMO: Nos últimos 40 anos, o perfil mundial do câncer vem mudando. Encarado inicialmente como uma doença dos países ricos, nota-se atualmente que a maior parte de seu ônus global provêm de países de poucos recursos ou em desenvolvimento. Nas últimas décadas o câncer se tornou um problema de saúde pública mundial, com estimativas alarmantes para as décadas subseqüentes. No Brasil, as estimativas para o ano de 2012 serão válidas também para o ano de 2013 e revelam a ocorrência de aproximadamente 518.510 casos novos de câncer, incluindo os casos de pele não melanoma. São esperados um total de 257.870 casos novos para o sexo masculino. A grande questão é que cerca de $10 \%$ destes casos serão na faixa etária inferior a 45 anos e 1\% abaixo de 20 anos de idade. Embora o tratamento atual do câncer tenha aumentado consideravelmente as taxas de sobrevida em 5 anos de pacientes jovens, ele quase que invariavelmente estará associado a enorme risco de infertilidade, levando a impacto negativo importante na vida destes jovens homens. Este artigo de revisão aborda os mais atuais conceitos em preservação de fertilidade em homens jovens tratados de câncer, o crescimento deste campo dentro da oncologia e da urologia e o futuro deste tema tão relevante para nossa população. Até hoje existe uma importante desconexão entre os guidelines mundiais e a prática médica, quando o assunto é preservação da fertilidade em homens com câncer em idade reprodutiva.
\end{abstract}

DESCRITORES: Neoplasias/quimioterapia; Preservação da fertilidade; Fertilidade/efeitos de drogas; Homem; Adulto.
ABSTRACT: Over the past 40 years, the global profile of cancer is changing. Initially regarded as a disease of the rich countries, it is noted that currently most of its global burden comes from poor or developing countries. In recent decades cancer has become a public health problem worldwide, with alarming estimates for subsequent decades. In Brazil, the estimates for the year 2012 will be valid for the year 2013 and reveals the occurrence of approximately 518,510 new cases of cancer, including cases of non-melanoma skin. Are expected a total of 257,870 new cases for males. The great thing is that about $10 \%$ of these cases are in the age group below 45 years and $1 \%$ below 20 years of age. Although the current treatment of cancer has increased considerably survival rates at 5 years young patients, it is almost invariably associated with great risk of infertility, leading to significant negative impact in the lives of these young men. This review article discusses the most current concepts in preserving fertility in young men treated for cancer, the growth of this field within the oncology and urology and the future of this topic so relevant to our population. Even today there is a important disconnect between the world and medical practice guidelines, when it comes to fertility preservation in men with cancer of reproductive age

KEYWORDS: Neoplasms/drug therapy; Fertility preservation; Fertility/drug effects; Men; Adult.

Trabalho foi realizado na Divisão de Urologia do HC-FMUSP. Setor de Reprodução Humana e Infertilidade Masculina. 1. Urologista, médico assistente do Hospital das Clínicas da Faculdade de Medicina da Universidade de São Paulo HC-FMUSP.

2. Oncologista, Hospital Israelita Albert Einstein - SP.

3. Professor Titular da Divisão de Urologia da Faculdade de Medicina da Universidade de São Paulo - FMUSP.

Endereço para correspondência: Rua Oscar Freire, 2250, Unidades T9/T10 Jardim Paulista. São Paulo, SP, CEP: 05409011. E-mail: conradoalvarenga@ hotmail.com. drconradoalvarenga @ hotmail.com 
Alvarenga $\mathrm{C}$ et al. Preservação da fertilidade em homens jovens com câncer.

\section{INTRODUÇÃO}

Nas últimas décadas o câncer se tornou um problema de saúde pública mundial, com estimativas alarmantes para as décadas subseqüentes. No Brasil, as estimativas para o ano de 2012 serão válidas também para o ano de 2013 e revelam a ocorrência de aproximadamente 518.510 casos novos de câncer, sendo que mais da metade serão no sexo masculino'.

A grande questão é que cerca de $10 \%$ destes casos serão na faixa etária inferior a 45 anos e 1\% abaixo de 20 anos de idade. Com os avanços nas terapias oncológicas, a sobrevida média e livre de doença em 5 anos vem aumentando consideravelmente e o comprometimento da fertilidade destes jovens homens surgindo como um tema relevante dentro do espectro de qualidade de vida após a cura da doença².

Desta forma, a preservação da fertilidade emerge como um tema de forte relevância atualmente. Cerca de $95 \%$ dos oncologistas americanos relatam abordar o aspecto da fertilidade com seus pacientes jovens mas apenas $50 \%$ deles encaminham este grupo especial de pacientes para um especialista em medicina reprodutiva ${ }^{3}$.

Uma das mais importantes considerações ao se determinar qual será o tratamento que o paciente será submetido, principalmente para adolescentes e homens jovens que não tiveram filhos e ainda os desejam ter, é: qual será o prejuízo da fertilidade após a recuperação e a busca pela cura?

Um estudo recente e bastante interessante revelou que mais de $50 \%$ dos homens jovens com câncer demonstram desejo de paternidade futuro, mas apenas $24 \%$ deles tinham sêmen congelado em banco antes de iniciar o tratamento. Acredita-se que barreiras sócio-econômicas, religiosas e culturais também dificultem a consolidação da percepção de obrigatoriedade de se ter sêmen congelado antes de se iniciar qualquer tipo de tratamento oncológico com risco em potencial ${ }^{4}$. Além disso, a falta de conhecimento de todos os profissionais que lidam com o doente oncológico jovem colabora negativamente para as taxas tão baixas de estoque de sêmen em pacientes oncológicos. Os profissionais que lidam com o câncer precisam ter a noção clara da enorme importância de se discutir sempre o risco de infertilidade e a necessidade, na maioria das ocasiões, de se congelar sêmen em banco antes do inicio de qualquer tratamento oncológico. $\mathrm{O}$ arrependimento é sentimento unânime entre os homens jovens, tratados de câncer, que não foram esclarecidos adequadamente da possibilidade ou da necessidade de se ter amostra congelada em banco de sêmen, antes de iniciar o tratamento oncológico ${ }^{5}$.

Desta forma procuramos levantar os aspectos mais atuais do aconselhamento pré tratamento do câncer, com o foco em adultos jovens do sexo masculino, as estratégias para se otimizar a preservação da fertilidade neste grupo, os mecanismos para se buscar o restauro da fertilidade após o tratamento, o surgimento da onco-fertilidade nos Estados Unidos, sub-especialidade focada neste tema e o futuro da preservação da fertilidade, como o transplante de células germinativas, o enxerto de tecido testicular e o testículo artificial.

\section{Etiologia da infertilidade no câncer}

Existem inúmeros mecanismos pelos os quais o câncer pode afetar negativamente a fertilidade do homem ${ }^{6}$. A equipe multidisciplinar que lida com o câncer precisa ter a noção clara que tanto os efeitos diretos e indiretos da neoplasia primária como os efeitos dos tratamentos, sejam eles cirúrgicos, quimioterápicos, radioterápicos ou multimodais, podem abrigar a gênese da infertilidade pós câncer.

A carcinogênese por si só pode resultar em um estado inflamatório sistêmico e a produção aumentada de citocinas pode levar a dano direto no tecido germinativo testicular. Além disso, inúmeras neoplasias cursam com aumento da temperatura corpórea em geral, levando também comprometimento da espermatogênese. Em fases mais avançadas, o câncer se comporta como uma doença crônica que afeta diretamente o estado nutricional, podendo também ter efeito negativo na produção de espermatozóides ${ }^{7}$. Mais especificamente, as neoplasias podem alterar o equilíbrio hormonal, como por exemplo tumores testiculares produtores de hCG, que por feedback negativo diminuem a produção de $\mathrm{LH}$ e $\mathrm{GnRH}$ pelo eixo na diminuição da espermatogênese ${ }^{8}$. Portanto, fica claro que o câncer inicialmente já pode ser um importante responsável pelo comprometimento da fertilidade masculina, mesmo que nenhum tratamento tenha sido ainda instituído. Estudos interessantes em homens portadores de Linfoma de Hodking, revelam piora da qualidade seminal em $70 \%$ dos casos no momento do diagnóstico, durante o exame realizado antes do congelamento seminal.8f Desta forma, o comprometimento da fertilidade relacionado ao câncer é multifatorial e mesmo antes do início de qualquer tratamento já pode haver impacto direto na qualidade seminal apenas pela presença do câncer ${ }^{9}$.

\section{O tratamento cirúrgico do câncer e infertilidade}

Embora não seja aplicável a todos os tipos de 
neoplasias, a cirurgia é uma potencial causa de infertilidade durante o tratamento do câncer. Dependerá fundamentalmente do local da neoplasia primária e da extensão da ressecção cirúrgica. Cirurgias pélvicas extensas, sejam gastrointestinais ou urológicas, podem levar a impacto direto dos plexos simpáticos e parassimpáticos pélvicos, comprometendo diretamente os mecanismos de ereção e ejaculação e consequentemente a fertilidade. Frente a este risco, técnicas mais modernas e apuradas caminham no sentido de melhorar a preservação dos feixes nervosos, diminuir a extensão da ressecções e se utilizar de tecnologia mais moderna e precisa durante as cirurgias para o tratamento de tumores pélvicos, como por exemplo a cirurgia robótica ${ }^{10}$. No caso mais específico de tumores testiculares, a redução da concentração de espermatozóides no sêmen pode chegar a $70 \%$ nos homens submetidos a orquiectomia unilateral e até $10 \%$ de azoospermia, mesmo em lesões unilaterais ${ }^{11}$.

O futuro sem dúvida alguma trará o desenvolvimento de técnicas de ressecção mais focadas e menos extensas, com redução da morbidade e consequentemente do risco de infertilidade.

\section{Quimioterapia e infertilidade masculina}

O efeito da quimioterapia na fertilidade do homem depende diretamente do agente empregado no tratamento e na dosagem determinada ${ }^{12}$. O epitélio germinativo é extremamente sensível a determinadas classes de quimioterápicos, que levam a dano direto ao DNA e RNA, assim como a indução de apoptose ${ }^{13}$. As células de Leydig parecem ser menos sensíveis aos efeitos dos quimioterápicos, embora elevações de LH sejam detectadas em até $90 \%$ dos homens com linfoma de Hodking tratados com quimioterapia sistêmica ${ }^{14,15}$.

Duas classes de quimioterápicos estão classicamente ligadas a maior risco de infertilidade pós tratamento: são os agentes alquilantes (ciclofosfamida) e as platinas (cisplatina e carboplatina), embora antimetabólicos, alcalóides e os inibidores da topoisomerase também podem ter efeito gonadotóxico direto. atuando como coadjuvantes da infertilidade ${ }^{16}$.

\section{A radiação e comprometimento da fertilidade}

O epitélio germinativo é também bastante sensível aos efeitos da radiação ${ }^{17}$. O dano ao DNA ocorre em todas as células germinativas, mas com graus variados de sensibilidade, dependendo basicamente das taxas e velocidades de mitose. Doses maiores de radiação, administradas de maneira menos fraciona- das, parecem ter efeito menos danoso ao testículo, quando comparado com doses menores fracionadas em um período de tempo maior, mesmo que a dose total seja equivalente em ambos os tratamentos ${ }^{18}$. O mecanismo que justifique tais achados ainda não seja totalmente claro na literatura.

Inúmeros trabalhos abordam a relação entre dosagem de radiação e a conseqüência na espermatogênese. Azoospermia é notada por um período de 5 anos, em média, com dosagens entre 4-6 Gy (Gray), enquanto com dosagens menores que $1 \mathrm{~Gy}$, este quadro é mais transitório, com a azoospermia permanecendo em média por 9 a 18 meses $^{19}$. Em contrapartida, as células de Leydig parecem ser mais resistentes a radiação. Apenas doses superiores a 20-30 Gy são suficientes para gerar um quadro de hipogonadismo dependente de reposição hormonal. Doses menores que 20 Gy levam a hipogonadismo sub-clinico, apenas com elevação nos níveis de hormônio luteinizante (LH).

\section{Os tratamentos multimodais}

A grande maioria dos estudos que avaliam o impacto sobre a fertilidade após o tratamento do câncer estão inseridos em um contexto com tratamento multimodal, com múltiplos agentes quimioterápicos, associados frequentemente ao tratamento radioterápico, geralmente em neoplasias hematopoiéticas e testiculares. No caso dos tumores testiculares, é muito importante que o urologista e o oncologista abordem a questão da fertilidade obrigatoriamente, mesmo nos casos de tumores unilaterais que são tratados sem a necessidade de quimioterapia adjuvante. Estudos relacionados ao tratamento com Cisplatina revelam recuperação da espermatogênese de $80 \%$ em 5 anos. Quando a Cisplatina é substituída pela Carboplatina, os impactos na fertilidade são reduzidos. Estudo muito importante que avaliou pacientes com tumor testicular não seminomatoso, em estádio I de alto risco, que receberam quimioterapia baseada em Carboplatina, revelou taxas de recuperação de normospermia em $93 \%$ dos casos submetidos a dois ciclos e $83 \%$ nos que receberam quatro ciclos de quimioterapia ${ }^{20}$.

Sabe-se hoje que estes homens que recuperaram a função testicular após o tratamento terão descendentes com risco $17 \%$ maior de mal formações congênitas, independente se gerados por gravidez espontânea ou por técnicas de reprodução assistida $^{21}$.

\section{Importância do aconselhamento pré tratamento}

Todos os pacientes com diagnóstico de câncer 
Alvarenga $\mathrm{C}$ et al. Preservação da fertilidade em homens jovens com câncer.

e com risco de comprometimento da fertilidade devem ser avaliados como candidatos a técnicas de preservação da fertilidade antes do inicio do tratamento. Em estudo interessante publicado em 2002 no Journal of Clinical Oncology, 92\% dos médicos e profissionais que lidavam com câncer em homens jovens sabiam claramente da necessidade de se oferecer o congelamento do sêmen antes do inicio do tratamento oncológico, mas apenas $48 \%$ deles confirmaram que sempre iniciavam esta discussão com os pacientes no inicio do tratamento ${ }^{1}$.

Este trabalho serviu como alicerce para o surgimento das recomendações da Sociedade Americana de Oncologia Clínica em 2006 e da Sociedade Americana de Pediatria em 2008, que tornaram obrigatória, antes do tratamento oncológico, a oferta de criopreservação de sêmen para todos os homens em idade reprodutiva. Mesmo assim, as taxas de sêmen congelado em banco pelo mundo ainda são muito discrepantes quando comparadas ao número de homens em idade reprodutiva tratados anualmente com câncer. Acredita-se que menos de $25 \%$ deste grupo tenha sêmen em banco, sugerindo até hoje uma desconexão entre os múltiplos guidelines e a prática médica.

A fertilidade poderá ser recuperada espontaneamente após o tratamento, mas isto não ocorrerá em todos os homens e dificilmente poderemos predizer aqueles que serão afetados pela esterilidade definitiva. Desta forma, fica muito claro que todos os homens submetidos a terapias para o câncer merecem atenção especial quanto a possiblidade de estratégias de preservação da fertilidade ${ }^{22}$.

\section{Métodos de preservação da fertilidade}

Antes de entendermos com clareza os melhores métodos de preservação de fertilidade, é preciso que fiquem claro alguns pontos cruciais do processo de espermatogênese e suas consequências sobre a preservação da fertilidade, suas limitações e a busca por alternativas de preservação específicas para homens em fase pré-puberal. Durante o processo de reprodução assistida, a injeção intra-citoplasmatica de espermatozóides demanda a presença de células germinativas maduras, ou seja, não é possível realizar tal tipo de tratamento com espermatogônias e espermatócitos. A limitação para pacientes em fase pré-puberal, tratados de câncer, é que nesta fase do desenvolvimento os testículos possuem apenas espermatogônias e espermatócitos e portanto este grupo especial de pacientes não se beneficia do congelamento de sêmen para sua utilização futura em injeção intra-citoplasmática ${ }^{23}$. Portanto, a idade é fator crucial na determinação de como a preservação da fertilidade poderá ser realizada nos homens.

Para aqueles em fase pré-puberal, a criopreservação de tecido testicular pode ser oferecida em alguns centros específicos pelo mundo, mas ainda é considerada experimental. Como será discutido adiante, o potencial uso, no futuro, da maturação in-vitro de espermatogônias para espermatócitos, ou o transplante de células tronco germinativas para o tecido testicular, poderão ser opções nesta fase de vida do homem que será tratado de câncer.

Já para os homens em fase pós puberal, a criopreservação de semen permanece como o meIhor método de preservação da fertilidade. Levando em consideração que a maioria dos pacientes apresentam alterações na qualidade e quantidade de espermatozóides antes do tratamento, como discutido anteriormente, recomenda-se que duas ou três amostras sejam congeladas, com intervalo de pelo menos $48 \mathrm{~h}$ entre cada uma delas ${ }^{24}$. Em pacientes com limitações para utilização do sêmen ejaculado, outras formas de obtenção de espermatozóides poderão ser necessárias, principalmente nos casos de distúrbios ejaculatórios. A vibro-estimulação e a eletro-ejaculação são exemplos de técnicas para se obter espermatozóides em homens com anejaculação. Em alguns casos também pode-se obter amostras diretamente do epidídimo ou dos testículos, como por exemplo em homens vasectomizados previamente ${ }^{25}$. Com o advento de modernas técnicas de reprodução assistida, estes homens com sêmen congelado poderão se beneficiar de tal medida protetiva, mesmo que venham a se tornar azoospérmicos definitivos após o tratamento do câncer ${ }^{26}$.

\begin{tabular}{|l|l|l|l|}
\hline & Método estabelecido & \multicolumn{1}{|c|}{ Método experimental } & Tempo requerido \\
\hline Homens em fase pré-puberal & Nenhum & $\begin{array}{l}\text { Criopreservação de tecido } \\
\text { testicular }\end{array}$ & Mínimo um dia antes \\
\hline Homens em fase pós puberal & $\begin{array}{l}\text { Criopreservação de } \\
\text { sêmen }\end{array}$ & $\begin{array}{l}\text { Criopreservação de tecido } \\
\text { testicular }\end{array}$ & $\begin{array}{l}\text { Mínimo 3 amostras com } \\
\text { intervalo de 48h entre elas }\end{array}$ \\
\hline
\end{tabular}




\section{Futuro da preservação da fertilidade}

Com o desenvolvimento e crescimento das técnicas de reprodução assistida, a criopreservação de sêmen em homens adultos com câncer mantêm a possibilidade da paternidade biológica, mesmo que a azoospermia definitive seja o desfecho após o tratamento do câncer.

O futuro da preservação da fertilidade masculina caminha do sentido de romper uma importante barreira: a injeção intra-citoplasmática de espermatozóide requer células germinativas maduras, que não se desenvolvem antes do período da puberdade masculine ${ }^{26}$. Portanto o foco das pesquisas atuais $e$ futuras é a busca de alternativas de preservação em homens com câncer na fase vida pré-puberal ou na infância. Homens em fase pré-puberal apresentam apenas espermatogônias e espermatócitos nos testículos e portanto não se beneficiam de criopreservação de sêmen, pois não apresentam linhagens celulares maduras ${ }^{27}$.

O uso de células tronco germinativas (CTG) parece ser o campo mais promissor no futuro da preservação da fertilidade de homens com câncer em fase pré-puberal. As células tronco de espermatogônias são células germinativas muito específicas que se diferenciam para iniciar o processo que leva a formação do espermatozóide ${ }^{28}$. São células indiferenciadas, que possuem a capacidade de de auto-renovar e de se diferenciar em espermatozóides maduros. O grande desafio atual é que são células em número limitado, de difícil identificação e cultura, além de não conhecermos até o momento sua atividade biológica exata ${ }^{29}$. Até o presente momento, dois desafios ainda precisam ser solucionados: quais o sinais que desencadeiam e estimulam as CTG iniciarem o processo de diferenciação e quais o sinais que as fazem se dividir e formar novas CTG ${ }^{30}$.

Após o tratamento do câncer, com o uso de terapias gonadotóxicas, encontra-se nos testículos apenas células de Sertoli. Isto pode ser resultado da perda por completo das CTG ou da impossibilidade das células de Sertoli modularem a sua diferenciação ${ }^{31}$.

As perspectivas para o futuro envolvem a possibilidade de transplante de células tronco germinativas com o intuito de restauro da fertilidade. Como exemplo lúdico temos a possibilidade de transplante de células tronco da medula óssea para um favorável ambiente testicular e estas células se diferenciam em linhagens germinativas maduras. Outra possibilidade é que o processo de diferenciação no sentido de células germinativas maduras seja realizado em outros ambientes não testiculares, aumentando muito o leque de possibilidades de utilização das CTG, inclusive para o sexo feminine ${ }^{31}$.

Estudos mais completos em mamíferos ainda serão necessários, a melhor compreensão da criopreservação de tecido testicular em homens na fase pré-puberal e melhor capacidade de manipulação e uso das células tronco possibilitarão a formação de recurso renovável de células germinativas, não apenas para restauro da fertilidade mas também para o uso em inúmeras outras doenças.

\section{CONCLUSÃO}

Embora o diagnóstico e tratamento do câncer ainda possam ser desafiadores e mesmo com a melhora nítida dos resultados nas últimas décadas, muitos dos homens tratados em idade reprodutiva ainda não são corretamente informados a respeito da preservação da fertilidade e aconselhados de maneira adequada com relação a terapias preservação. O rápido encaminhamento destes doentes para um urologista especializado em medicina reprodutiva é essencial, pois algumas das estratégias de preservação de fertilidade necessitam de 2 a 3 semanas para serem concluídas. Muitas questões técnicas, logísticas e éticas acerca da preservação da fertilidade ainda estão em aprimoramento e o futuro fará com que este campo continue a crescer, até que fique sedimentado como etapa fundamental no tratamento do homem com câncer.

\section{REFERÊNCIAS}

1. Jani RJ, Dean EM, Charles CC. Fertility preservation. Maio Clin Proc. 2011;86(10:45-9.

2. American Cancer Society. Cancer in Children. Available from: http://www.cancer.org/cancer/cancerinchildren/detailedguide/cancer-in-children-treatingsurvival-rates.

3. Forman EJ, Anders CK, Behera MA. A nationwide

survey of oncologists regarding treatment-related in fertility preservation in female cancer patients. Fertil Steril. 2010;94:1652-6.

4. Leena N, Laurie EC, Richard NY. Barriers to fertility preservation in male adolescents with cancer: its time for a multidisciplinary approach that includes urologists. Urology. 2012;79(6):1206-9. 
Alvarenga $C$ et al. Preservação da fertilidade em homens jovens com câncer.

5. Achille MA, Rosberger Z, Robitaille R, et al. Facilitators and obstacles to sperm banking in young men receiving gonadotoxic chemotherapy for câncer: the perspective of survivors and health care professionals. Hum Reprod. 2006;21:3206-16.

6. Landon WT, Robert EB. Oncofertility and the male cancer patient. Cur Treat Option Oncol. 10.1007/ s11864-012-0191-7.

7. Sabanegh JES, Ragheb AM. Male fertility after cancer. Urology. 2009;73:225.

8. Rueffer U, Breuer K, Josting A, et al. Male gonadal dysfunction in patients with Hodgkins disease prior to treatment. An Oncol. 2001;12:1307.

9. Dohle GR. Male infertility in câncer patients: review of the literature. Int J Urol. 2010;17:327.

10. Foster RS, McNulty A, Rubin LR, et al. The fertility of patients with clinical stage I testis cancer managed by nerve sparing retroperitoneal lymph node dissection. J Urol. 1994;152:1139.

11. Petersen PM, Skakkebaek NE, Vistisen K, et al. Semen quality and reproductive hormones before orchiectomy in men with testicular cancer. J Clin Oncol. 1999;17:941.

12. Waxman J. Chemotherapy and the adult gonad: a review. J R Soc Med. 1983;76:144.

13. Hsiao W, Stahl PJ, Osterberg EC, Nejat E, Palermo GD, Rosenwaks Z, et al. Succesful treatment of postchemotherapy azoospermia with microsurgical testicular sperm extraction: the Weill Cornell experience. J Clin Oncol. 2011;29:1607-11.

14. Heikens J, Behrendt $\mathrm{H}$, Adriaanse R, Berghout A. Irreversible gonadal damage in male survivors of pediatric Hodgkins disease. Cancer. 1996;78:2020-4.

15. Bramswig JH, Heimes $U$, Heiermann E, Schlegel W, Nieschlag E, et al. The effects of different cumulative doses of chemotherapy on testicular function: results in 75 patients treated for Hodgkins disease during childhood or adolescence. Cancer. 1990;65:1298302.

16. Waxman J, Terry Y, Rees LH, et al. Gonadal function in men treated for acute leukaemia. Br Med J. 1983;287:1093.

17. Arserini $P$, Chiodi S, Spinelli S, Costa M, Conte N, Copello $F$, et al. Sêmen analysis following allogenic bone marrow transplantation. Additional data for evidence-based counseling. Bone Marrow Transplant.
2002;30:447-51

18. Ash $\mathrm{P}$. Influence of radiation on fertility in man. $\mathrm{Br} \mathrm{J}$ Radiol. 1980;15:271.

19. Sandeman TF. The effects of $x$ irradiation on male human fertility. Br J Radiol. 1966;39:901.

20. Pectasides D, Pectasides M, Farmakis D, et al. Testicular function in patients with testicular cancer treated with bleomycin-etoposide-carboplatin (BEC90) combination chemotherapy. Eur Urol. 2004;45:187.

21. Stahl O, Boyd HA, Giwercman A, Lindholm M, Jensen A, Kjaer SK,et al. Risk of birth abnormalities in the offspring of men with a history of câncer: a cohort study using Danish and Swedish national registries. J Natl Cancer Inst. 2011;103:398-406.

22. Pont J, Albretch W. Fertility after chemotherapy for testicular germ cell cancer. Fertil Steril. 1997;68:1-5.

23. Kazutoshi F, Hiroshi O, et al. Transplantation of spermatogonial stem cells isolated from leukemic mice restores fertility without inducing leukemia. J Clin Invest. 2005;115(7):1855-61.

24. Lee SJ, Schover LR, Partridge $\mathrm{AH}$, et al. American Society of Clinical Oncology recommendations on fertility preservation in cancer patients. J Clin Oncol. 2006;24:2917.

25. Muller J, Sonksen J, Soomer P, et al. Cryopreservation of semen from pubertal boys with cancer. Med Pediatr Oncol. 2000;34:191.

26. Agarwal A, et al. Fertility after cancer. A prospective review of assisted reproductive outcome with banked semen specimens. Fertil Steril. 2004;81:342-8.

27. Hellstrom WJG. Male infertility and sexual dysfunction. New York: Springer; 1997.

28. J. Zhang and L. Li. Stem cell niche: microenvironment and beyond. J Biol Chem. 2008;283(15):9499-503.

29. Mimeault M, Batra S. Concise review: recent advances on the significance of stem cells in tissue regeneration and cancer therapies. Stem Cells. 2006;24(11):231945.

30. Caíres K, Broady J, Maclean D. Maintaining the male germline: regulation of spermatogonial stem cells. J Endocrinol. 2010;205(2):133-45.

31. Shetty G, Meistrich ML. Hormonal aproaches to preservation and restoration of male fertility after cancer treatment. J Nat Cancer Inst. 2005;34:18794. 